\title{
Taking a closer look at PD pathology
}

Shahmoradian
et al. found
that the
principal
components
of most of
the inclusions
appeared to
be crowded
membranous
material,
as well as
distorted
vesicular
structures
and cellular
organelles

Lewy bodies and Lewy neurites are intraneuronal inclusions that are considered key neuropathological features of Parkinson disease (PD). It has been hypothesized that a fibrillar, or amyloid, form of $\alpha$-synuclein - a protein implicated in PD pathogenesis - is the core component of these structures. However, in a new study, Shahmoradian et al. report that $\alpha$-synuclein-immunopositive inclusions in tissue from individuals with PD largely comprise crowded membranes and organelles.

The role of Lewy bodies and Lewy neurites in the pathogenesis of PD remains unclear, as does how these structures form. To obtain a better understanding of their make-up, and hence to gain insight into the potential mechanisms underlying PD, Shahmoradian et al. adopted a correlative light and electron microscopy approach.

In this approach, alternate sections of post-mortem PD tissue (no more than $450 \mathrm{~nm}$ apart) were histologically stained for $\alpha$-synuclein and assessed by light microscopy

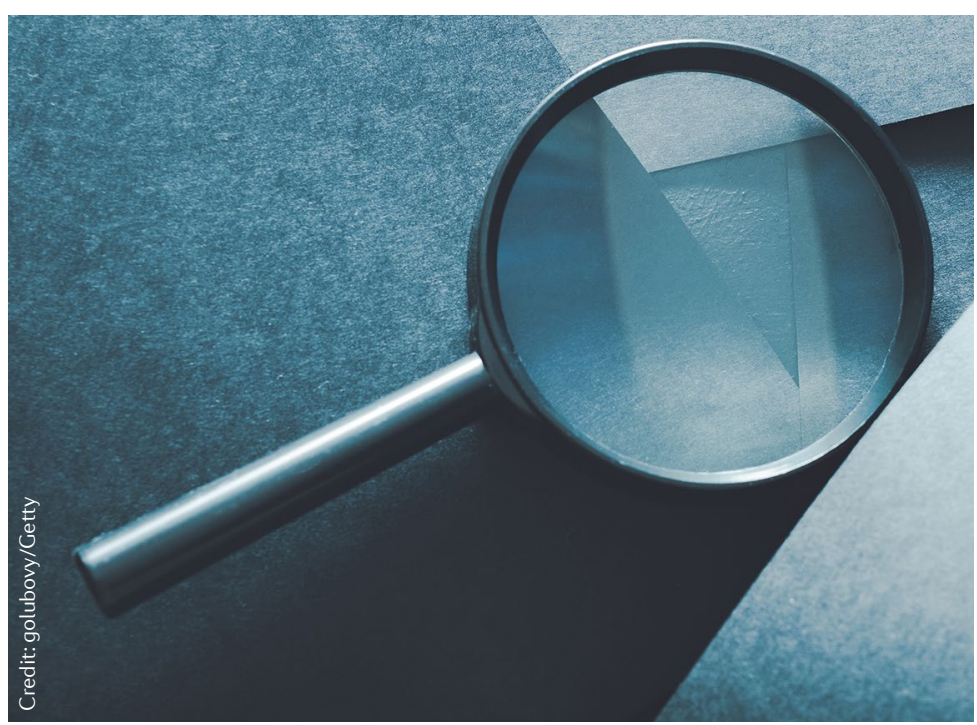

or prepared for use in transmission electron microscopy (TEM). Through the adoption of this approach, the authors obtained 3D ultrastructural views of 17 a-synuclein-immunopositive inclusions - both Lewy bodies and Lewy neurites - from the CA2 region of the hippocampus or the substantia nigra from five individuals with PD.

The authors detected filamentous structures in many of the inclusions. However, only three of the inclusions primarily consisted of such filaments and only one of the inclusions, which was in the substantia nigra, consisted of a proteinacious core, with radiating filaments and peripheral organelles.

Strikingly, Shahmoradian et al. found that the principal components of most of the inclusions appeared to be crowded membranous material, as well as distorted vesicular structures and cellular organelles. Indeed, in addition to identifying mitochondria at the periphery and centre of some of the inclusions, the authors also detected L-shaped structures, which they concluded were reminiscent of lysosomes and autophagosomes. Further assessment of tissue from one of the brain donors by serial block-face scanning electron microscopy (SBFSEM) revealed similar findings.

Next, the authors used stimulated emission depletion microscopy (STED) and markers for mitochondria and lysosomes to more closely probe the identity of the vesicular structures in Lewy pathology in tissue from 14 brain donors. In line with the findings from the TEM and SBFSEM experiments, STED revealed the presence of organelles - including lysosomes and mitochondria and remnants of them in different a-synuclein-immunopositive intracellular inclusions.

In the final phase of the study, Shahmoradian et al. directly confirmed the high level of lipid content in the Lewy pathology. After showing co-staining of lipids and $\alpha$-synuclein in tissue from the substantia nigra and the CA2 by confocal microscopy, they used anti-Stokes Raman scattering, Fourier transform infrared spectroscopic imaging and lipidomics to confirm the lipid-rich nature of the inclusions.

Together, these findings indicate that Lewy bodies and Lewy neurites are often rich in membranous material and challenge the view that they are primarily proteinacious inclusions. Moreover, according to the authors, these data provide support for the hypothesis that impairment in the intracellular trafficking of organelles has a role in the pathogenesis of PD.

Darran Yates

ORIGINAL ARTICLE Shahmoradian, S. H. et al. Lewy pathology in Parkinson's disease consists of crowded organelles and lipid membranes. Nat. Neurosci. 22, 1099-1109 (2019) 